\title{
LA ACTIVIDAD APÍCOLA EN LA REGIÓN DE MURCIA
}

\author{
POR \\ CAYETANO ESPEJO MARÍN
}

\section{Introducción}

La apicultura ha constituido una actividad de marcada importancia en la economía agraria murciana desde tiempos muy lejanos. La tradición apícola en la Región de Murcia tiene un lógico desarrollo si tenemos en cuenta la abundante variedad de frutales y productos de huerta con los que cuenta. La existencia de esta gran variedad de frutales de hueso, cítricos y otras variedades agrícolas, además del espacio ocupado por el monte, unido al buen clima que se da, hacen de la Región un lugar idóneo para la apicultura, consiguiéndose excelentes calidades de mieles, principal producto obtenido del cuidado y cría de las abejas.

En la actualidad ya nadie pone en duda la labor polinizadora de las abejas. Es de sobra conocido que es uno de los mejores agentes polinizadores y que en determinados cultivos, como es el caso del almendro, la existencia o no de abejas influye considerablemente en la producción final.

Se da la paradoja de que mientras en países como Estados Unidos, Argentina, Gran Bretaña, Países Bajos, Japón y otros, los agricultores arriendan las colmenas durante el período de floración de

Cayetano Espejo Marín. Departemento Geografía Física, Humana y Análisis Regional. Universidad de Murcia.

Estudios Geográficos

Tomo LVII, n. ${ }^{\circ}$ 224, julio-septiembre 1996 
árboles frutales y de ciertas plantas forrajeras, en nuestra Región, son los apicultores los que tienen que pagar en dinero o en especies por asentar sus colmenas en fincas o montes (López, 1983).

Los productos más conocidos de las abejas son sobre todo la miel y la cera, pero también hay que tener en cuenta el polen, la jalea real y el propóleo. El polen adquiere cada día mayor importancia como producto comercial apícola para su utilización cosmética, farmacológica, alimentación humana, etc. En nuestra Región la obtención de polen para su comercialización es pequeña y solamente la llevan a cabo un reducido número de apicultores, aunque cada día aumenta su obtención por el incremento de su importancia y utilización.

La miel es el producto más conocido y generalizado y por tanto el que aporta los mayores beneficios al apicultor. En la miel se distinguen distintas variedades según la flor de la que ha sido obtenida. Cada variedad posee características diferentes y por tanto calidad y precios diferentes.

En la Región de Murcia son numerosas las variedades de miel que existen. Cuatro son las más conocidas y comercializadas: de azahar, de romero, de espliego y de mil flores. Las mieles de azahar y de romero son muy apreciadas en el mercado nacional, siendo Murcia una zona privilegiada para su obtención, por la abundancia de montes y de grandes zonas de cultivos de cítricos.

La mayoría de los apicultores murcianos se localizan en la comarca del Noroeste, por la tradición existente y por ser una zona con abundante superficie de monte. La segunda comarca con elevado censo de apicultores es la Vega del Segura, espacio con importantes extensiones de cítricos, con una floración de azahar muy importante.

El alcance de la apicultura murciana en el conjunto nacional viene dado por dos hechos. Primero, la Región de Murcia, según el Anuario de Estadística Agraria de 1990, es la quinta provincia en cuanto al número de colmenas, detrás de Valencia, Cáceres, Badajoz y Salamanca, y en segundo lugar, en producción de miel es la tercera provincia, con $1.360 \mathrm{Tm}$, cantidad superada solamente por Valencia y Badajoz, que producen 4.269 y $1.854 \mathrm{Tm}$ de miel respectivamente. 


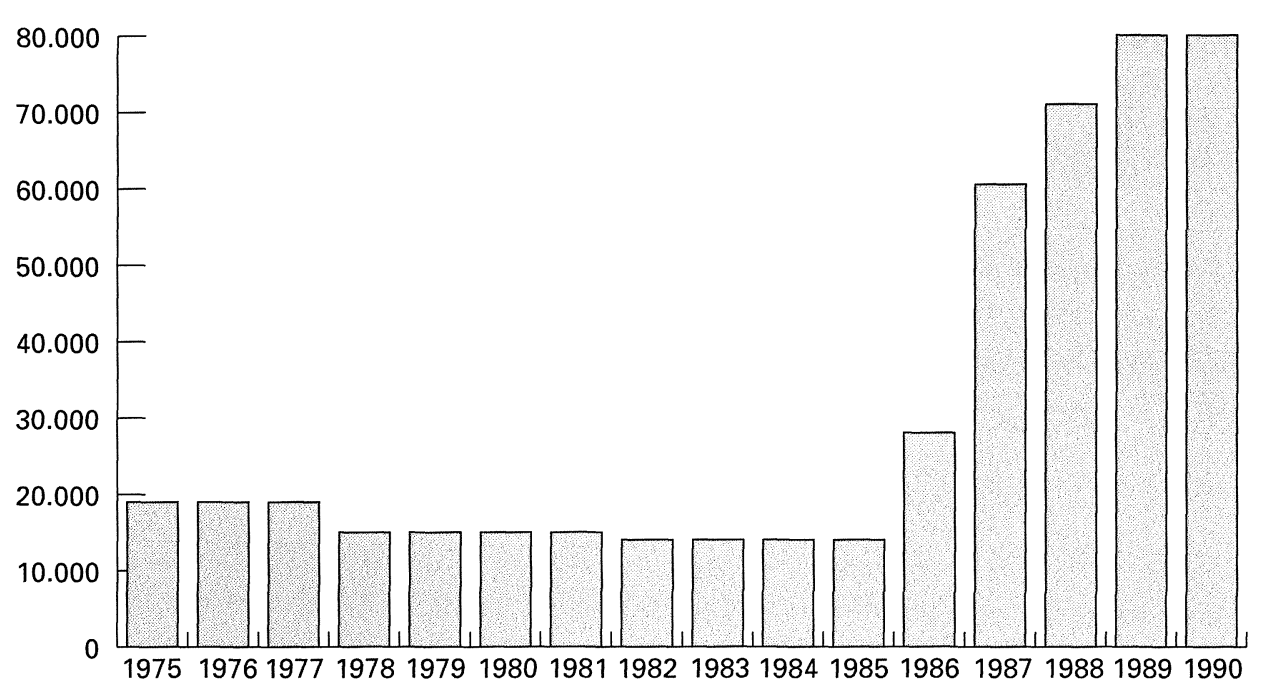

Figura 1.-Evolución del número de colmenas en la Región de Murcia. 1975-1990

\section{Evolución de la apicultura murciana}

La evolución de la apicultura en la Región de Murcia durante los últimos quince años viene caracterizada sobre todo por la estabilidad del censo de colmenas (fig. 1). La aparición en el verano de 1985 de la Varroa ${ }^{1}$ da lugar a que se produzcan dos hechos: por un lado la muerte de bastantes colmenas, y en segundo lugar la mayoría de los apicultores declaran sus colmenas ante las posibles ayudas que pueden recibir de la Administración Regional. Esto sirve para que se ponga al día, lo más aproximado, el censo regional de colmenas, de ahí que se refleje en la Figura 1 un crecimiento muy elevado, cuando en realidad no es tan grande. El censo de colmenas de la Región de Murcia durante la segunda mitad de los años ochenta crece un poco, motivado sobre todo por la concienciación de los agricultores, en especial los almendricultores, en el tema de la polinización como factor muy importante para la producción. También influye el

1 Ácaro que se introduce en la celdilla de cría cuando la abeja está en estado de ninfa y se alimenta del producto que la abeja lleva a la celdilla para alimento de la cría, que nace defectuosa por falta de nutrición. (Writter, 1989). En la actualidad la Varroa está totalmente controlada en la Región de Murcia. 
«boom» ecologista y que los apicultores profesionales tienen cada vez mayor número de colmenas para hacer rentable su profesión y la comercialización de su propia producción. Durante los años 1989 y 1990 se mantiene estabilizado el número de colmenas, debido a la caída, iniciada años atrás, de los precios de la miel.

Con los datos del Censo Ganadero de 1974 y el Listado Apícola Regional de 1990 se puede conocer cuál ha sido la evolución a nivel municipal del número de colmenas en la Región de Murcia (fig. 2).

Un primer aspecto a destacar es que mientras de las 18.576 colmenas censadas en 1974, 4.329 son fijistas, lo que significa el $23 \%$ del total, las 33.594 contabilizadas en 1990 son todas movilistas, debido sobre todo a razones sanitarias, ya que las fijistas no se pueden curar cuando han sido afectadas por la Varroa.

De la observación de la figura 2 se desprende como la mayoría de los municipios han incrementado su número de colmenas. Merecen especial atención aquellos en los que han crecido las colmenas en más de mil durante el período de 1974 a 1990: Yecla, Calasparra, Cieza, Murcia y Cartagena. Otros municipios, que apenas si tienen colmenas en 1974, cuando se compara la situación con el año 1990 se detectan también incrementos muy grandes, como sucede con Pliego, Cieza, Molina de Segura, Alcantarilla, Aguilas y Totana, que al menos triplican su censo de colmenas durante esta etapa.

Las colmenas reducen su número en sólo seis municipios: Bullas, Campos del Río, Aledo, Mazarrón, Alhama y Librilla. Todos, salvo Bullas, con un número inferior a 100 en 1974, por lo que la bajada no incide en el total regional, frente a los incrementos tan importantes reseñados más arriba.

\section{Distribución de la apicultura}

La apicultura es una actividad que se da en la mayoría de los municipios de la Región de Murcia, tal y como queda reflejado en la figura 3 , en la que se expone la distribución municipal de las colmenas y explotaciones apícolas.

Dos son las zonas de la Región en las que se sitúan la mayoría de las colmenas: la mitad septentrional del espacio regional y la Vega 


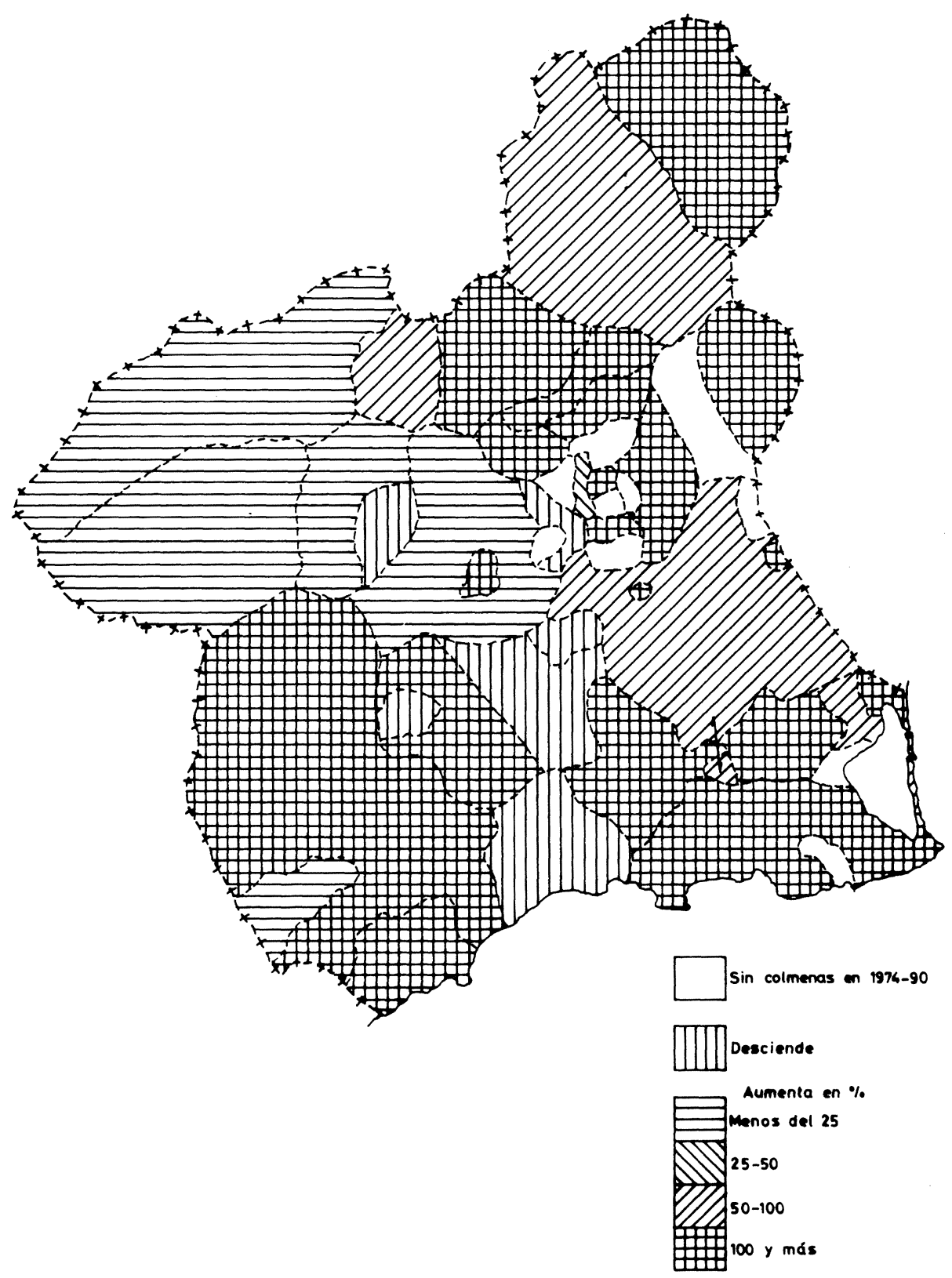

Fuente: Consejería de Agricultura

Figura 2.-Evolución municipal del número de colmenas. 1974-1990 


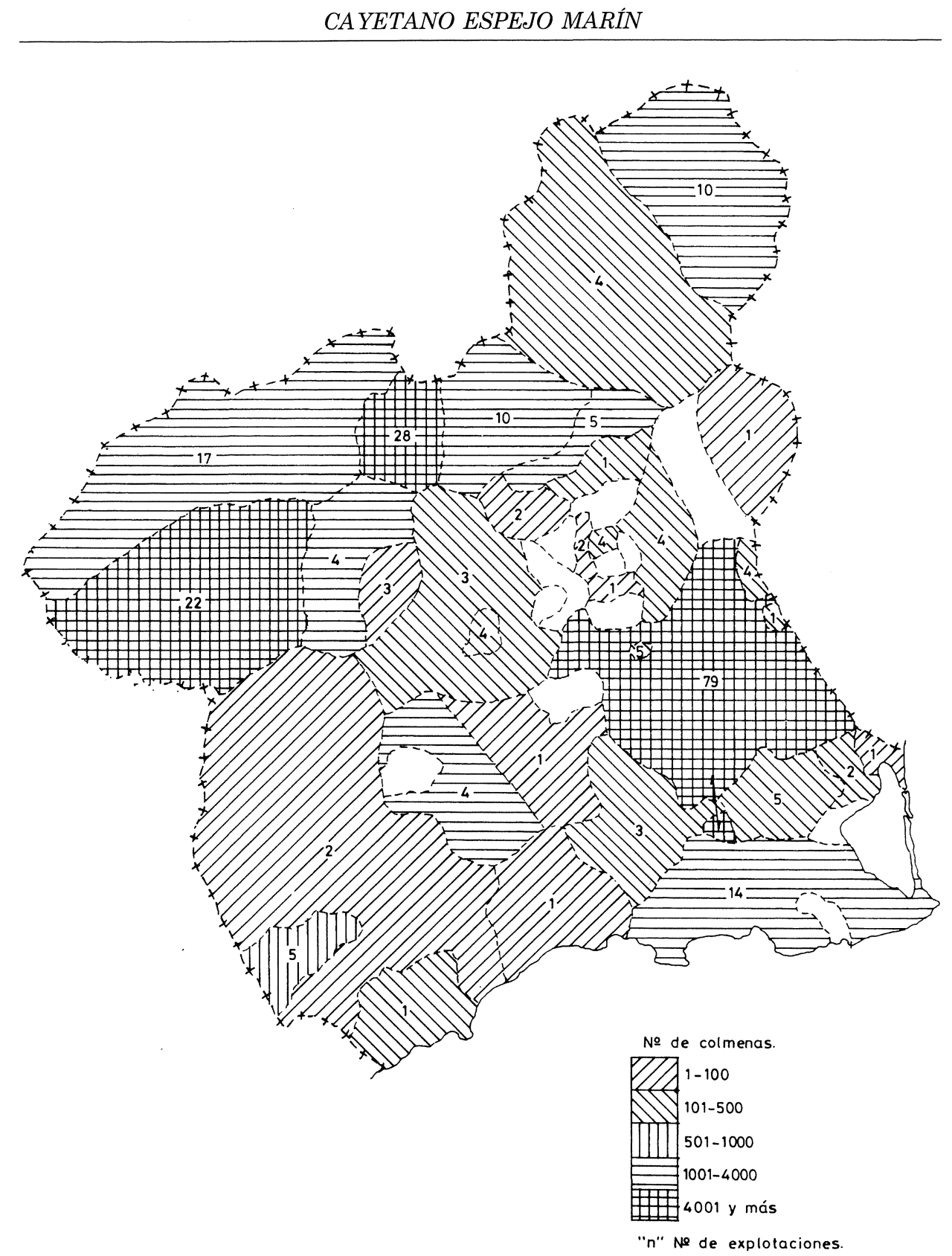

Fuente: Consejeria de Agricultura

Figura 3.-Distribución de la Apicultura Murciana. 1990

$$
-458-
$$


del Segura, debido a que se trata de una zona con abundante superficie montañosa, en el primer caso, y en la Vega del Segura por la abundancia de cítricos.

La mayor parte de las explotaciones y colmenas se localizan en unos pocos municipios (cuadro I). Murcia cuenta con el $30 \%$ de explotaciones y el $22 \%$ de las colmenas censadas en la Región. Le sigue en importancia Calasparra, con el $11 \%$ de explotaciones y el $15 \%$ de las colmenas. El tercer municipio con un aporte destacable al total regional es Caravaca, que acumula el $8 \%$ de las explotaciones y el $12 \%$ de las colmenas. Los tres municipios suman cerca de la mitad de las explotaciones y de las colmenas censadas en 1990 en la Región de Murcia.

Otros municipios con un número que supera las mil son Abarán, Cartagena, Cehegín, Cieza, Moratalla, Totana y Yecla. En otros municipios la apicultura presenta un carácter testimonial, ya que cuentan con un número de colmenas que no llega a las cien, como sucede con Abanilla, Alguazas, Alhama, Bullas, Lorca, Mazarrón, Ricote, San Pedro del Pinatar y Villanueva del Río Segura.

La media de colmenas por explotación presenta oscilaciones muy fuertes de unos municipios a otros, tal y como se puede observar en el cuadro I. Merecen especial atención los casos de Blanca, Cehegín, Totana y Cieza que presentan una media superior a 295 colmenas/ explotación. A continuación se encuentra un grupo de municipios cuya media de colmenas/explotación gira en torno a las doscientas, como son Abarán, Beniel, Calasparra y Caravaca. Frente a esta situación, en otros se da una media inferior a 50 colmenas/explotación: Aguilas, Alhama, Bullas, Lorca, Mazarrón, Ricote, Torre Pacheco y Villanueva del río Segura.

De lo analizado se desprende que quizás sea la tradición la que determina la mayor o menor incidencia de la apicultura en unas zonas determinadas, quedando la influencia de los factores físicos y humanos en la mayoría de los casos un tanto al margen, ya que lo que en un área tiene una relación directa, en otra, con una circunstancias similares, apenas si tiene incidencia.

En general la presencia de colmenas en la Región de Murcia coincide con zonas de montaña donde existen importantes extensiones de 


\section{Cuadro I}

DISTRIBUCIÓN MUNICIPAL DE LAS COLMENASY EXPLOTACIONES APÍCOLAS EN LA REGIÓN DE MURCIA 1990

\begin{tabular}{|c|c|c|c|c|c|}
\hline & \multicolumn{2}{|c|}{ EXPLOTACIONES } & \multicolumn{2}{|c|}{ COLMENAS } & \multirow{2}{*}{$\begin{array}{l}\text { COLMENAS/ } \\
\text { EXPLOTACION }\end{array}$} \\
\hline & Número & $\%$ & Número & $\%$ & \\
\hline ABANILLA & 1 & 0,38 & 50 & 0,15 & 50 \\
\hline ABARAN & 5 & 1,89 & 1.105 & 3,29 & 221 \\
\hline AGUILAS & 12 & 4,55 & 300 & 0,89 & 25 \\
\hline ALCANTARILLA & 5 & 1,89 & 407 & 1,21 & 81 \\
\hline ALGUAZAS & 1 & 0,38 & 65 & 0,19 & 65 \\
\hline ALHAMA & 1 & 0,38 & 3 & 0,01 & 3 \\
\hline ARCHENA & 4 & 1,52 & 491 & 1,46 & 123 \\
\hline BENIEL & 1 & 0,38 & 200 & 0,60 & 200 \\
\hline BLANCA & 1 & 0,38 & 500 & 1,49 & 500 \\
\hline BULLAS & 3 & 1,14 & 70 & 0,21 & 23 \\
\hline CALASPARRA & 28 & 10,61 & 5.019 & 14,94 & 179 \\
\hline CARAVACA & 22 & 8,33 & 4.046 & 12,04 & 184 \\
\hline CARTAGENA & 14 & 5,30 & 1.294 & 3,85 & 92 \\
\hline CEHEGIN & 4 & 1,52 & 1.578 & 4,70 & 395 \\
\hline CIEZA & 10 & 3,79 & 2.954 & 8,79 & 295 \\
\hline FUENTE ALAMO & 3 & 1,14 & 230 & 0,68 & 77 \\
\hline JUMILLA & 4 & 1,52 & 455 & 1,35 & 114 \\
\hline LORCA & 2 & 0,76 & 42 & 0,13 & 21 \\
\hline MAZARRON & 1 & 0,38 & 20 & 0,06 & 20 \\
\hline MOLINA DE SEGURA & 4 & 1,52 & 490 & 1,46 & 123 \\
\hline MORATALLA & 17 & 6,44 & 1.696 & 5,05 & 100 \\
\hline MULA & 3 & 1,14 & 490 & 1,46 & 163 \\
\hline MURCIA & 79 & 29,92 & 7.335 & 21,83 & 93 \\
\hline PLIEGO & 4 & 1,52 & 320 & 0,95 & 80 \\
\hline PUERTO LUMBRERAS & 5 & 1,89 & 675 & 2,01 & 135 \\
\hline RICOTE & 2 & 0,76 & 80 & 0,24 & 40 \\
\hline SAN JAVIER & 2 & 0,76 & 190 & 0,57 & 95 \\
\hline SAN PEDRO & 1 & 0,38 & 50 & 0,15 & 50 \\
\hline SANTOMERA & 4 & 1,52 & 410 & 1,22 & 103 \\
\hline TORRE PACHECO & 5 & 1,89 & 125 & 0,37 & 25 \\
\hline TOTANA & 4 & 1,52 & 1.292 & 3,85 & 323 \\
\hline VILLANUEVA & 2 & 0,76 & 80 & 0,24 & 40 \\
\hline YECLA & 10 & 3,79 & 1.532 & 4,56 & 153 \\
\hline TOTAL & 264 & 100 & 33.594 & 100 & 127 \\
\hline
\end{tabular}

Fuente: Consejería de Agricultura. Listado de Registro Apícola.

$$
-460-
$$


matorral aromático, como sucede con la comarca del Noroeste, en la que la tradición apícola representa un papel de primer orden, y en los municipios de Cieza, Abarán, Jumilla y Yecla. Estos dos últimos presentan grandes superficies de secanos extensivos con cultivos poco rentables. Si a ello unimos en muchos casos que se trata de explotaciones agrarias con escasa base territorial, y por tanto insuficientes, se explica el que los agricultores busquen ingresos adicionales a través de la apicultura. Otra zona con abundantes colmenas es la Vega del Segura, área en la que la abundancia de cítricos y de frutales de hueso hace que se produzcan importantes floraciones de aprovechamiento tradicional, como sucede con el azahar.

En conjunto las floraciones aprovechadas en cada comarca son:

- Altiplano: romero, matorral y almendro.

- Noroeste: romero, almendro, espliego y frutales de hueso.

- Vega del Segura: cítricos, alfalfa, matorral, frutales de hueso y cultivos de verano.

- Valle del Guadalentín: almendro, algodón y cultivos de verano.

- Campo de Cartagena: almendro, cítricos, matorral y cultivos de verano.

Concluyendo cabe afirmar, coincidiendo con Segrelles (1989), que la influencia de los factores señalados sobre la apicultura es relativa, ya que la mayoría de los apicultores, sobre todo los profesionales y los que comparten la apicultura con otra actividad agraria o pecuaria, practican la trashumancia, volviendo en invierno a sus lugares de origen para aprovechar la vegetación, los cultivos autóctonos y un clima benigno.

\section{Tipos de colmenas}

En España se ha practicado la apicultura con colmenas de corcho hasta hace relativamente pocos años. Fue hacia 1940 cuando en Valencia se inició el cambio a la colmena movilista «Layens». En Extremadura este cambio no se realizó hasta el año 1960 aproximadamente, y en Salamanca ocurrió aún más tarde (Peris Martínez, 1984). En 
la Región de Murcia se introduce la colmena Layens a mediados de los años sesenta.

El corcho es una colmena peón fijista formada por un cilindro de corteza de alcornoque que lleva varas (trencas) como refuerzos. Los panales (bresca) los realiza libremente la colonia y son de forma irregular, quedando fijos al cuerpo con propóleos. La parte central de los panales está ocupada por la cría (el nido) mientras que las partes superior e inferior, así como los panales laterales están ocupados por la miel. Cuando se practicaba esta apicultura los traslados de las colmenas se solían hacer con caballerías y los recipientes utilizados para contener la miel eran de cuero o pellejos de los empleados para el aceite y el vino.

Las colmenas fijistas o colmenas «corchos» antes eran numerosas en algunas zonas de la Región, como Águilas, pero en la actualidad solamente quedan algunas aisladas. La mayoría han desaparecido por razones sanitarias, ya que la varroa da lugar a que este tipo de colmenas, al no poderse curar, desaparezcan. Además la colmena fijista presenta una producción de miel menor que la movilista.

Los tipos de colmenas que manejan los apicultores de la Región de Murcia son de disposición horizontal, tipo Layens, de 10 a 12 cuadros de $30 \times 35 \mathrm{~cm}$, colmena que se puede decir que solamente se trabaja en España, y con más arraigo en la zona de Levante y Murcia. Este tipo de colmena movilista horizontal pervive, según los apicultores, por sus ventajas para la trashumancia. Tiene un manejo sencillo y necesita una menor inversión, pero con ella se obtienen bajos rendimientos (18-20 kg en un año bueno) y menos proporción de núcleos monoflorales, ya que es menos productiva y menos higiénica que la colmena en alzas o de disposición vertical, de la que sólo se utilizan en la Región unas 8.000 , el $10 \%$ del total, pero su número crece en detrimento de la tipo Layens. El rendimiento medio de la colmena en alza oscila entre los 25 y $30 \mathrm{~kg}$ de miel al año.

Este escaso número de colmenas en alzas se debe a que el apicultor sólo se ha interesado por la producción de miel en cantidad, sin mostrar demasiado interés por la obtención de mieles monoflorales, de gran aceptación en países como Alemania o Inglaterra, lo que da una idea del escaso nivel profesional de la mayoría de los apicultores murcianos. 
La abeja española es la «Apis Mellífera» o Abeja Negra, que tiene una gran actividad fuera de la colmena, es decir en el pecoreo. Esta abeja es muy sensible, y por esta razón resulta muy agresiva. Tiene mucha tendencia a la emjambrazón ${ }^{1}$, esto se ve favorecido por el desarrollo de la colmena en alza, que permite ir añadiendo tantas alzas como sean necesarias, sin necesidad de desplazamiento de la colmena.

\section{Estructura de las explotaciones}

Dos son las fuentes que se utilizan para la elaboración de este apartado. El Censo Agrario, que nos ofrece la actividad apícola según la estructura de las explotaciones agrarias sobre las que se asientan. Esta información se encuentra muy infravalorada, pues el número de colmenas que ofrece el Censo Agrario de 1989 es de 15.093, más de cinco veces inferior a los datos que proporciona la Estadística Agraria Regional, elaborada por la Consejería de Agricultura, y que según esta fuente ascienden a 80.000. Estas distorsiones son debidas a la confección de los Censos Agrarios. Sin embargo, el Censo Agrario nos aporta la distribución cualitativa y territorial del número de explotaciones, además de que es la única fuente que nos ayuda a conocer la relación entre apicultura y soporte agrícola.

La otra fuente estadística utilizada es el Listado de Registro Apícola de 1990, elaborado por la Consejería de Agricultura. En él se da una relación con el nombre del apicultor, localidad de residencia y número de colmenas que posee. Esta fuente pese a ofrecer también un número de colmenas inferior a las existentes en la realidad, 35.600 frente a las 80.000 citadas con anterioridad, es un instrumento muy fiable, ya que se realiza con la información que dan los apicultores al darse de alta en el Registro Regional de Explotaciones Apícolas, sobre todo por la subvención que reciben de la Consejería de Agricultura, y que hasta 1991 asciende a 500 pesetas por colmena. El Listado de Registro Apícola es la única fuente que nos permite

2 Consiste en una forma de dispersión de la especie apícola, haciendo que los colectivos de estos individuos con posibilidades reproductoras y de supervivencia se alejen de la colmena original (Puerta y otros, 1989). 
analizar la estructura de las explotaciones según el número de colmenas que poseen y por tanto la categoría profesional de los apicultores, coincidiendo en este caso la estructura de las explotaciones con la propiedad de las colmenas, premisa que no es común en todas las especies ganaderas.

La actividad apicola según la estructura de las explotaciones agrarias

La estructura de las explotaciones con ganado apícola entre 1962 y 1989 ha conocido apreciables cambios como se puede observar en el cuadro II, en el que se describe la situación según la superficie de las explotaciones en los años 1962, 1982 y 1989, ya que el Censo Agrario de 1972 no contabiliza las colmenas.

Durante las tres últimas décadas se ha producido un fortísimo descenso del número de explotaciones, que en este caso coincide con el de propiedades (fig. 4). La reducción ha supuesto una división de las explotaciones por un índice de 6,6, una disminución del número de explotaciones en 990, lo que representa el $85 \%$ menos de las censadas en 1989 respecto a 1962. En cuanto a tamaño, las mayores de 5 ha son las que presentan una reducción más acusada, y dentro de este grupo las situadas en los intervalos de 10 a 30 ha, que pasan de 331 en 1962 a 29 en 1989, y las de 30 a 100 ha que de 194 en 1962 pasan a ser 16 en 1989, lo que supone para ambos tipos de explotaciones un descenso del $92 \%$.

En cambio, durante este período se ha duplicado el número de colmenas, lo que ha dado lugar a que se produzca un incremento considerable del número de colmenas por explotación, pasando la media regional de 6,2 a 85,7 entre el Censo Agrario de 1962 y el de 1989, siendo en 1962 las explotaciones sin tierras las que tienen mayor número de colmenas/explotación, igual que sucede en 1989 (fig. 5). Son los apicultores profesionales que practican la trashumancia y aprovechan las distintas floraciones bien dentro del espacio regional o en otras provincias, de ahí que no precisen tener tierras propias para desarrollar esta actividad. 
Cuadro II

ESTRUCTURA DE LAS EXPLOTACIONES CON GANADO APÍCOLA EN LA REGIÓN DE MURCIA. 1962-1989

\begin{tabular}{|c|c|c|c|c|c|}
\hline & \multicolumn{2}{|c|}{$\begin{array}{r}1962 \\
\text { EXPLOTACIONES }\end{array}$} & \multicolumn{2}{|c|}{ COLMENAS } & \multirow{2}{*}{$\begin{array}{l}\text { COLMENAS/ } \\
\text { EXPLOTACION }\end{array}$} \\
\hline & Número & $\%$ & Número & $\%$ & \\
\hline Sin tierras & 106 & 9,09 & 2.386 & 32,72 & 22,51 \\
\hline$<1$ ha & 82 & 7,03 & 691 & 9,48 & 8,43 \\
\hline $1-5 \mathrm{ha}$ & 230 & 19,73 & 1.203 & 16,50 & 5,23 \\
\hline 5-10 has & 160 & 13,72 & 687 & 9,42 & 4,29 \\
\hline $10-30$ has & 331 & 28,39 & 1.061 & 14,55 & 3,21 \\
\hline $30-100$ has & 194 & 16,64 & 781 & 10,71 & 4,03 \\
\hline \multirow[t]{2}{*}{100 y más has } & 63 & 5,40 & 483 & 6,62 & 7,67 \\
\hline & 1.166 & 100 & 7.292 & 100 & 6,25 \\
\hline \multicolumn{6}{|c|}{1982} \\
\hline & \multicolumn{2}{|c|}{ EXPLOTACIONES } & \multicolumn{2}{|c|}{ COLMENAS } & \multirow{2}{*}{$\begin{array}{l}\text { COLMENAS/ } \\
\text { EXPLOTACION }\end{array}$} \\
\hline & Número & $\%$ & Número & $\%$ & \\
\hline Sin tierras & 27 & 8,08 & 2.835 & 26,67 & 105,00 \\
\hline$<1$ ha & 55 & 16,47 & 2.024 & 19,04 & 36,80 \\
\hline $1-5$ has & 78 & 23,35 & 2.739 & 25,76 & 35,12 \\
\hline $5-10$ has & 47 & 14,07 & 1.026 & 9,65 & 21,83 \\
\hline 10-30 has & 64 & 19,16 & 1.582 & 14,88 & 24,72 \\
\hline $30-100$ has & 41 & 12,28 & 275 & 2,59 & 6,71 \\
\hline 100 y más ha & 22 & 6,59 & 150 & 1,41 & 6,82 \\
\hline \multirow[t]{4}{*}{ TOTAL } & 334 & 100 & 10.631 & 100 & 31,83 \\
\hline & \multicolumn{4}{|c|}{1989} & \multirow{3}{*}{$\begin{array}{l}\text { COLMENAS/ } \\
\text { EXPLOTACION }\end{array}$} \\
\hline & \multicolumn{2}{|c|}{ EXPLOTACIONES } & \multicolumn{2}{|c|}{ COLMENAS } & \\
\hline & Número & $\%$ & Número & $\%$ & \\
\hline Sin tierras & 27 & 15,34 & 6.977 & 46,23 & 258,41 \\
\hline$<1$ ha & 43 & 24,43 & 3.503 & 23,21 & 81,47 \\
\hline $1-5$ has & 38 & 21,59 & 2.322 & 15,38 & 61,11 \\
\hline $5-10$ has & 15 & 8,52 & 708 & 4,69 & 47,20 \\
\hline 10-30 has & 29 & 16,48 & 687 & 4,55 & 23,69 \\
\hline $30-100$ has & 16 & 9,09 & 769 & 5,10 & 48,06 \\
\hline 100 y más has & 8 & 4,55 & 127 & 0,84 & 15,88 \\
\hline TOTAL & 176 & 100 & 15.093 & 100 & 85,76 \\
\hline
\end{tabular}

Fuente: I.N.E. Censos Agrarios. 


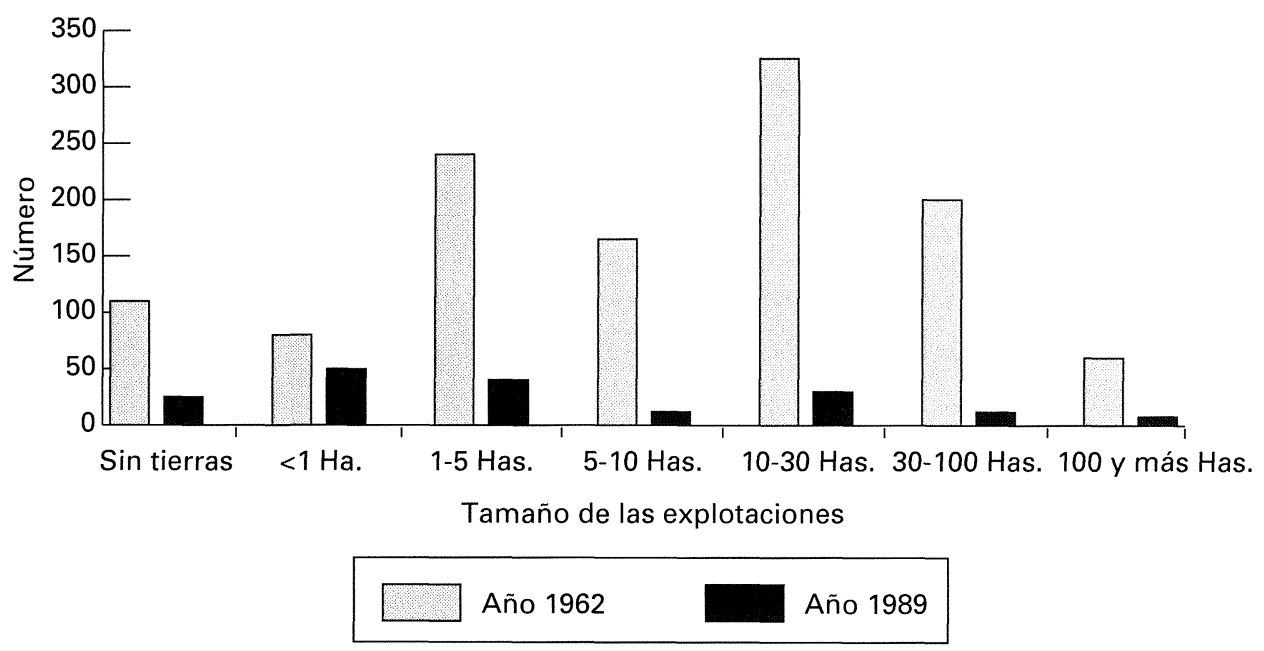

Figura 4.-Evolución del número de explotaciones con ganado apicola. 1962-1989

Clasificación de los apicultores en función de las colmenas que poseen

La Región de Murcia cuenta con un censo de 80.000 colmenas, repartidas de un modo desigual entre unos 300 apicultores, lo que da una media de 267 colmenas/explotación. La única fuente que nos permite acercarnos al estudio de la estructura de las explotaciones apícolas según el número de colmenas que poseen, es el Listado de Registro Apícola elaborado por la Consejería de Agricultura. A pesar de la gran diferencia entre el número de colmenas existente en la Región, 80.000, y el número que nos da el citado listado, 33.594, éste nos permite conocer la categoría de la mayoría de los apicultores murcianos. En función del número de colmenas que poseen se pueden clasificar en tres grupos:

a) Apicultores profesionales. Se dedican exclusivamente a la apicultura. Tienen 400 y más colmenas, y son el $7 \%$ del total de los apicultores, pero poseen el $42 \%$ de las colmenas (fig. 6). Suelen realizar entre 3 y 5 salidas trashumantes al año con unas programaciones muy intensivas y con un acentuado carácter industrial. Este tipo de 


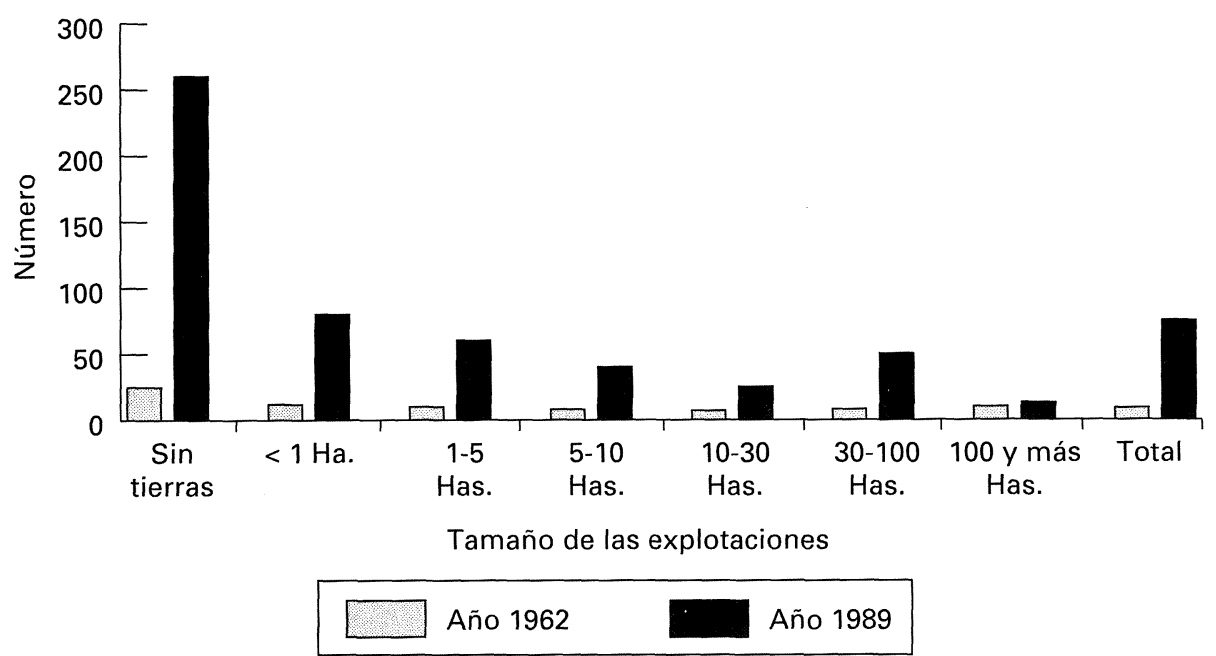

Figura 5.-Número medio de colmenas por explotación. 1962 y 1989

apicultores se localizan preferentemente en unos pocos municipios de las comarcas del Noroeste y de la Vega del Segura (cuadro III).

b) Apicultores semiprofesionales. Este grupo está compuesto por los apicultores que reciben aproximadamente la mitad de sus ingresos de la apicultura y el resto de la actividad agrícola o ganadera. Poseen entre 100 y 400 colmenas y constituyen una cuarta parte del total de los apicultores. Son propietarios del $35 \%$ de las colmenas. Realizan las mismas trashumancias que los profesionales. Los apicultores semiprofesionales se reparten por varios municipios de los que tienen colmenas en el espacio regional (cuadro III), pero destacan los municipios de Murcia y Calasparra que con 20 y 12 apicultores cada uno, suponen el 30 y el $18 \%$ respectivamente del total de los apicultores de este tipo que existen en la Región de Murcia.

c) Apicultores aficionados. Cuentan con un número inferior a 100 colmenas. Son los más numerosos, el $68 \%$, pero sólo suman el $22 \%$ de las colmenas de la Región. Practican una apicultura muy simple, sin trashumancia, o a veces realizan alguna salida en busca de una determinada floración. En este grupo se incluyen algunos apicultores que utilizan la colmena para la polinización de sus plantacio- 


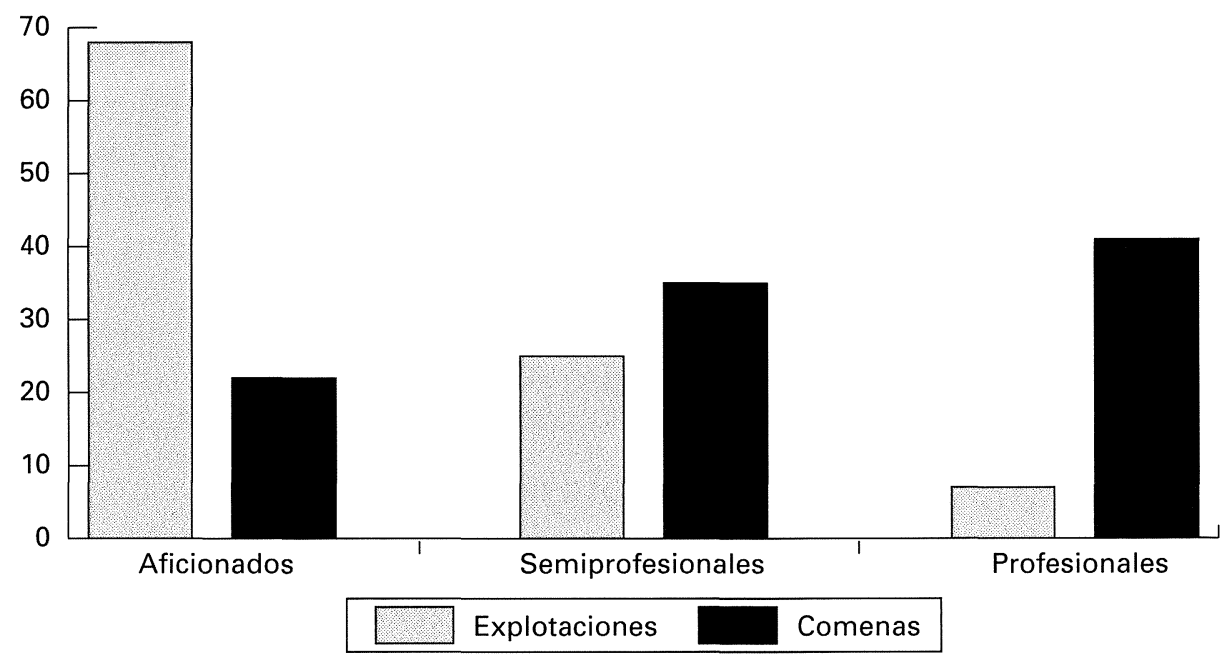

Figura 6.-Distribución de las explotaciones y colmenas según la categoría profesional de los apicultores. 1990

nes y al tiempo la miel obtenida sirve de complemento económico para la explotación. En muchos casos mantienen las colmenas por afición, consumiendo toda la producción, y para ellos la apicultura pasa de ser una afición a una «devoción». Los apicultores aficionados se reparten por toda la Región, no obstante, más de una cuarta parte de ellos se sitúa en el municipio de Murcia (cuadro III).

\section{Rutas apicolas trashumantes}

La Región de Murcia, pese a la abundancia de superficie con que cuenta para aprovechamiento apícola, es insuficiente para los apicultores que obtienen gran parte o la totalidad de sus ingresos de la apicultura, debido a que las floraciones que dan gran producción de miel aparecen en épocas muy concretas. Así la floración del almendro se da en los meses de enero y febrero, la del azahar en abril y mayo, y la del romero en octubre, noviembre y diciembre. Por ello los apicultores recurren a trashumancia para continuar la obtención de miel y otros productos.

La ruta que se usa con más frecuencia es la floración del girasol. Durante la segunda quincena de junio las colmenas se llevan a las 


$$
\text { CUADRo III }
$$

ESTRUCTURA DE LAS EXPLOTACIONES APÍCOLAS

DE LA REGIÓN DE MURCIA. 1990

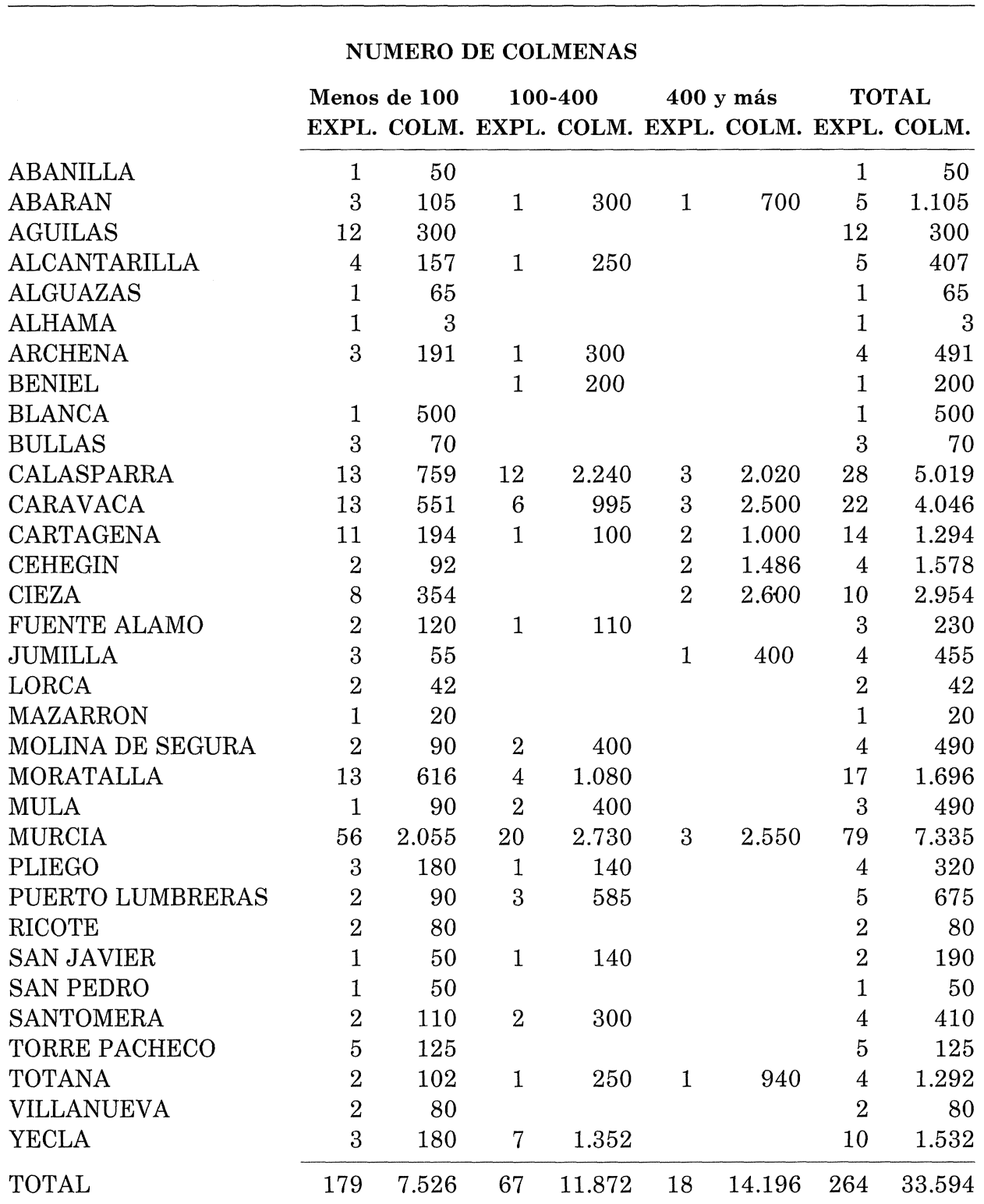

Fuente: Consejería de Agricultura. Listado de Registro Apícola.

$$
-469-
$$


provincias de Córdoba y Sevilla. El girasol acaba a mediados de julio en estas provincias y las colmenas se trasladan a Albacete y Cuenca para el aprovechamiento del girasol, que es más tardío que en Andalucía. A Albacete igualmente se desplazan con frecuencia para el aprovechamiento del romero durante los meses de noviembre y diciembre.

Cuando acaba el girasol las colmenas se transportan a las sierras de Jaén y Granada con el fin de aprovechar el espliego y otras plantas aromáticas.

Con menor frecuencia se realiza la trashumancia a las provincias de Salamanca, Cáceres y Badajoz, donde varía el producto obtenido, así del brezo obtienen miel hacia el mes de marzo, de la encina mielatos en el mes de mayo, y en este mes de la jara aprovechan su polen.

Un escaso número de apicultores trasladan sus colmenas a las provincias de Soria y Burgos donde aprovechan excelentes floraciones de cantueso y brezo durante los meses de marzo y abril.

Hasta hace poco eran frecuentes los desplazamientos a la provincia de Huelva para aprovechar las dos floraciones del eucalipto (junio-julio y octubre-noviembre).

La Región de Murcia también es utilizada por los apicultores valencianos y alicantinos que trasladan sus colmenas a comienzos de la primavera a los regadíos murcianos de limoneros, donde aprovechan fundamentalmente la floración del azahar (Segrelles, 1989).

\section{Conclusiones}

Como conclusión cabe apuntar que la Región de Murcia cuenta con unas excelentes condiciones para el desarrollo de la apicultura, debido a la abundancia de montes con matorral y de cultivos de cítricos, de frutales de hueso y de almendros.

Durante los últimos años se ha producido un crecimiento importante del número de colmenas, debido sobre todo al boom ecologista y a la cada vez mayor profesionalización de los apicultores. Estos han 
introducido un nuevo tipo de colmena, denominada en alzas, que les permite obtener mayor cantidad de miel que con la Layens.

La actividad apícola se desarrolla. sobre todo en explotaciones con escaso soporte territorial, y dentro de ella se dan tres tipos de clasificación de los apicultores en función del número de colmenas que poseen: aficionados, semiprofesionales y profesionales. Los dos últimos practican la trashumancia con el fin de hacer rentable su trabajo.

\section{BIBLIOGRAFÍA}

LÓPEZ, C. (1983): «La apicultura en la Región de Murcia», ONE, núm. 34, pp. 102-104.

Peris Martínez, J. (1984): «Producción y comercio de los productos apícolas en España», El Campo, núm. 93, pp. 40-68.

PueRTA, F., y otros (1989): «El fenómeno del enjambrazón en "Apis Mellifera“», en Curso de Apicultura, Madrid, Ministerio de Agricultura, Pesca y Alimentación, pp. 7981.

Segrelles SerRano, J. A. (1989): «La apicultura valenciana: un aprovechamiento agrario tradicional», Cuadernos de Geografía, núm. 45, pp. 73-88.

WritTER, W. (1989): «La Varroasis: Epizootiología y tratamiento», en Curso de Apicultura, Madrid, Ministerio de Agricultura, Pesca y Alimentación, pp. 113-121.

RESUMEN: La Región de Murcia cuenta con unas excelentes condiciones para el desarrollo de la apicultura, debido a la abundancia de matorral, y de frutales y cítricos. La actividad apícola se desarrolla sobre todo en explotaciones con escaso soporte territorial, por lo que es frecuente la trashumancia por parte de los apicultores profesionales.

Palabras Clave. Apicultura, Región de Murcia, abeja, colmena.

RÉSUMÉ. La région de Murcie dispose d'une série d'excellentes conditions favorisant le développement de l'apiculture, à cause de l'abondance de buissons, d'arbres fruitiers et d'agrumes. L'activité apicole se déroule surtout dans des exploitations à faible etendue, ce qui entraîne l'existence d'une transhumance fréquente chez les apiculteurs professionnels.

Mots Clé. Apiculture, Région de Murcie, abeille, ruche.

ABSTRACT. The region of Murcia has excellent conditions for the development of the beekeeping owing to the abundance of thicket, fruit trees and citrics. The beekeeping usually takes place in small operations with a limited territorial support; becuase of that, the transhumance is usual among professional beekeepers.

KEY Words. Beekeeping, Region of Murcia, bee, beehive. 